\title{
Longitudinal spin fluctuations in bec and liquid Fe at high temperature and pressure calculated with a supercell approach
}

\author{
Davide Gambino $\odot,{ }^{*}$ Marian Arale Brännvall, Amanda Ehn, Ylva Hedström, and Björn Alling \\ Department of Physics, Chemistry, and Biology (IFM), Linköping University, SE-581 83, Linköping, Sweden
}

(Received 17 April 2020; revised 4 June 2020; accepted 11 June 2020; published 2 July 2020)

\begin{abstract}
Investigating magnetic materials at realistic conditions with first-principles methods is a challenging task due to the interplay of vibrational and magnetic degrees of freedom. The most difficult contribution to include in simulations is represented by the longitudinal magnetic degrees of freedom [longitudinal spin fluctuation (LSF)] due to their inherent many-body nature; nonetheless, schemes that enable to take into account this effect on a semiclassical level have been proposed and employed in the investigation of magnetic systems. However, assessment of the effect of vibrations on LSF is lacking in the literature. For this reason, in this work we develop a supercell approach within the framework of constrained density functional theory to calculate self-consistently the size of local-environment-dependent magnetic moments in the paramagnetic, high-temperature state in the presence of lattice vibrations and for liquid $\mathrm{Fe}$ in different conditions. First, we consider the case of bcc Fe at the Curie temperature and ambient pressure. Then, we perform a similar analysis on bcc Fe at Earth's inner-core conditions, and we find that LSFs stabilize nonzero moments which affect atomic forces and the electronic density of states of the system. Finally, we employ the present scheme on liquid Fe at the melting point at ambient pressure and at Earth's outer-core conditions ( $p \approx 200 \mathrm{GPa}, T \approx 6000 \mathrm{~K}$ ). In both cases, we obtain local magnetic moments of sizes comparable to the solid-state counterparts.
\end{abstract}

DOI: 10.1103/PhysRevB.102.014402

\section{INTRODUCTION}

Magnetic materials find widespread application in many technological sectors, not only for their obvious magnetic properties [1] but also, for instance, as structural materials [2,3], the best example of which is steels. Since modern society needs always more efficient devices, the design of materials has recently started to be guided by theoretical calculations [4,5]. From this point of view, magnetism in solids poses a great challenge for modeling because of its many-body quantum nature and the difficulty in accurately representing thermal excitations, and for these reasons it has been and still is the subject of thorough theoretical investigations [6-8].

Solid-state magnetism is historically described within two limits: the localized moments and the itinerant electron models [9]. The former model, expressed in terms of a Heisenberg Hamiltonian, appropriately describes magnetic insulators since the electrons responsible for magnetic effects are here localized in the atoms and give rise to robust magnetic moments, which interact with each other through different

*davide.gambino@liu.se

Published by the American Physical Society under the terms of the Creative Commons Attribution 4.0 International license. Further distribution of this work must maintain attribution to the author(s) and the published article's title, journal citation, and DOI. Funded by Bibsam. types of exchange mechanisms. The latter, instead, refers to magnetic metals, where the electrons are delocalized, and their cooperative interaction gives rise to magnetic properties; this type of system has usually been investigated in terms of band ferromagnetism. These models are extremes of reality, and real systems fall in between these two classes.

A phenomenological theory that interpolates between the two limits was developed by Moriya [7] and takes as the fundamental variable the spin fluctuations. Its application to real systems was not widespread, although all subsequent theories in this field are based on it. Recently, a dynamical version of Moryia's spin fluctuation theory has been established [8] and applied to simple systems like bcc Fe and $\mathrm{Ni}$ [10], which seems to give quantitatively good results, although overestimating the Curie temperature.

To date, the only method that can account for magnetic effects in realistic systems of moderately large size is based on the Heisenberg model with parameters calculated from density functional theory (DFT). Theories that can take into account finite-temperature quantum excitations in magnetic materials, such as dynamical mean-field theory, are still confined to small system sizes [11], rarely involving more than a unit cell [12].

The original Heisenberg model describes magnetic moments of constant size on a fixed lattice that interact with each other through well-defined and constant exchange interactions. It can be employed in the investigation of more itinerant systems if one introduces more flexibility in the Hamiltonian: for a ferromagnetic metal, for instance, the exchange interactions end up being dependent on the local atomic and 
magnetic environment [13-16]; therefore, the introduction of distance-dependent and magnetic-state-dependent exchange interactions is needed in order to match experimentally available properties such as low-temperature spin-wave excitations [17] and the Curie temperature [13]. One issue here is related to the modeling of the paramagnetic phase in DFT calculations, but this can be achieved with the use of the disordered local moment (DLM) approach in one of its different flavors $[18,19]$. In particular, here, we work in the framework of the magnetic sampling method (MSM) [19], in which many different random magnetic configurations are employed in the calculations, and properties of the paramagnetic state are then retrieved as statistical averages. Within this method, one can also include magnetic short-range-order effects to model finite temperature magnetism with the aid of constrained DFT calculations.

One further benefit of the MSM method is that it enables simulations which consider vibrational and magnetic degrees of freedom (DOFs) on a similar footing, the first example of which is given by the disordered local moment molecular dynamics (DLM-MD) method [20]. A further development of this concept, inspired by spin-lattice dynamics simulations, is the atomistic spin dynamics combined with ab initio molecular dynamics (ASD-AIMD) approach [21], where interatomic forces are calculated within DFT and the time evolution of the moments is propagated in parallel according to the LandauLifshitz-Gilbert (LLG) equation, with the two dynamics communicating with each other the positions of the atoms and the direction of the spins, which determine the pair exchange interactions (and therefore the evolution of the moments) and the interatomic forces (and therefore the movement of the atoms), respectively.

In this type of simulation, only transversal spin fluctuations have been included so far [21], neglecting longitudinal spin fluctuations (LSFs); these are excitations along the direction of the magnetic moment that are not directly included in the standard Heisenberg Hamiltonian, and they are more intimately connected to the quantum nature of electrons compared to transversal fluctuations. Regular DFT calculations do not reproduce this effect because of the independent-particle nature of the Kohn-Sham electrons. Nonetheless, semiclassical models to include LSFs on a mean-field basis have been developed in recent years [7,22-30].

From the work of Murata and Doniach [22], in which a fourth-order dependence of the energy functional on the magnetic moment, inspired by the Ginzburg-Landau expansion, was introduced, many applications of this semiclassical approach have been performed on more or less itinerant systems. Uhl and Kübler [23] have applied this concept to bcc Fe, fcc $\mathrm{Ni}$, and fcc and hep Co by calculating all parameters of the model Hamiltonian with $a b$ initio calculations of spin spirals, considering fluctuations in reciprocal space. Similarly, Rosengaard and Johansson [24] investigated finite-temperature magnetism of the same systems but with a real-space formulation of the problem and employing the calculated parameters in Monte Carlo (MC) simulations to derive properties at and above the Curie temperature; for bcc Fe, they found a weakly increasing local magnetic moment as a function of temperature. Ruban et al. [25] investigated LSFs with DLM in the coherent-potential-approximation framework with a slight modification of the Heisenberg-Landau Hamiltonian and performed MC simulations based on these parameters calculated from first principles, finding a decrease in size of the average magnetic moment for bcc Fe. Wysocki et al. [27] studied more generally the thermodynamics of itinerant magnets in a classical spin fluctuation model, focusing on the problems related to the phase space measure (PSM). More recently, analytical expressions for the magnetic entropy were introduced in the single-particle potentials of DFT calculations in order to approximately include LSFs in calculations [31,32]. With this method, Dong et al. [28] calculated the thermal expansion in Fe based on a Debye-Grüneisen model.

Despite the rich literature, no direct investigation of the interplay between lattice vibrations and LSF can be found at the level of semiclassical theory. Dynamical mean-field theory calculations of vibrational properties can be found in the literature [33,34]; however, these studies focused solely on the effect of magnetism on phonon frequencies. The only attempt to calculate the reciprocal effect of phonons and spin fluctuations was performed with dynamic spin fluctuation theory [35] and found little effect of phonons on the magnetic properties of $\mathrm{Fe}$ (although in this method some experimental parameters are employed as the input of the calculations).

For this reason, in this work we develop a method to calculate the on-site energy landscape that governs LSFs and self-consistently calculate the magnetic moment at a given temperature for each atom in a vibrating lattice. Since LSFs are more important in the paramagnetic state, we focus only on this regime. We test the method on a snapshot taken from an ASD-AIMD simulation of bec Fe at its Curie temperature and on bcc Fe at Earth's inner-core conditions, employing a snapshot taken from a nonmagnetic (NM) MD run [36]. In addition, we investigate also liquid $\mathrm{Fe}$ at ambient pressure and around the melting point, and at conditions expected in Earth's outer core ( $p \approx 200 \mathrm{GPa}, T \approx 6000 \mathrm{~K})$, also in this case taking a single snapshot from MD simulations. We observe that the local environment strongly affects the shape of the on-site energy landscape, and the inclusion of LSFs in the calculation affects pressure and forces in the considered snapshot.

This paper is structured as follows: In Sec. II the semiclassical theory of LSFs is reviewed (Sec. II A), with special attention given to the issue of PSM, and the scheme for the calculation of the on-site energy and determination of the size of magnetic moments at a given temperature in a vibrating lattice is presented (Sec. II B), with computational details and the origin of the snapshots presented in Sec. IIC. In Sec. III A the scheme is tested on bcc Fe at ambient pressure and temperature close to the Curie temperature, and the effect of vibrations and magnetic disorder is inferred, together with an evaluation of the effect of different PSMs on the size of the magnetic moments. The application of the method on bcc Fe at Earth's inner-core conditions is then presented in Sec. III B, and results are compared with previous investigations. Section IIIC deals with liquid $\mathrm{Fe}$ at the melting point under ambient-pressure conditions and liquid $\mathrm{Fe}$ at Earth's outer-core conditions. Finally, in Sec. IV the conclusions of the work are summarized. 


\section{THEORETICAL METHODS}

\section{A. Semiclassical theory of longitudinal spin fluctuations}

For a system of $N$ magnetic moments, a generalized Heisenberg Hamiltonian can be written as

$$
H=-\sum_{i \neq j} J_{i j} \mathbf{m}_{i} \cdot \mathbf{m}_{j}+\sum_{i} E_{i}\left(m_{i}\right),
$$

where $J_{i j}$ is the exchange interaction between moment $\mathbf{m}_{i}$ and moment $\mathbf{m}_{j}$ and $E_{i}\left(m_{i}\right)$ is the on-site term that depends on the size of the moment $m_{i}$; bold symbols represent vectors here.

In general, both $J_{i j}$ and $E_{i}\left(m_{i}\right)$ can depend on the local arrangement of the atoms and the relative magnetic moments, with possible variations in these quantities as a function of the surrounding environment, depending on the nature of the system under investigation. The last term in Eq. (1) represents the energy of moment $\mathbf{m}_{i}$, immersed in the mean field created by all the other magnetic moments, as a function of its own magnitude: This is the semiclassical term associated with LSFs. For a system with very well localized magnetic moments, this term does not depend on the surrounding moments and has typically a sharp minimum, but for a more itinerant system the magnetic state plays a crucial role [25]. Its general form is inspired by Ginzburg-Landau thermodynamic theory of magnetism:

$$
E_{i}\left(m_{i}\right)=\sum_{n=0}^{\infty} a_{n} m_{i}^{2 n} \approx a m_{i}^{2}+b m_{i}^{4},
$$

where at least a fourth-order polynomial is needed to approximate a localized moment landscape.

Thermodynamic quantities for the Hamiltonian in Eq. (1) can be derived from the partition function:

$$
Z=\int d \mathbf{m}_{1} d \mathbf{m}_{2} \cdots d \mathbf{m}_{N} e^{-\frac{H}{k_{B} T}},
$$

where $k_{B}$ is the Boltzmann constant, $T$ is the temperature, and the integration extends to all possible configurations of the moments $\mathbf{m}_{i}$. In the paramagnetic state, the first term on the right-hand side of Eq. (1) is equal to zero; therefore, since the on-site term does not depend explicitly on other moments, the partition function can be written as the product of $N$ partition functions $Z=\prod Z_{i}$, one for each moment $\mathbf{m}_{i}$, with the single-moment partition function $Z_{i}$ defined as

$$
Z_{i}=\int d \mathbf{m}_{i} e^{-\frac{E_{i}\left(m_{i}\right)}{k_{B} T}}=\int_{0}^{\infty} d m_{i} \operatorname{PSM} e^{-\frac{E_{i}\left(m_{i}\right)}{k_{B} T}} .
$$

Here, the last expression is obtained with a change of variables from cartesian to spherical coordinates and integrating out the angular ones since the energy $E_{i}$ does not depend explicitly on them in the paramagnetic state in this approximation. Constants have been dropped because they cancel out or add an irrelevant constant shift to thermodynamic quantities. PSM is the phase space measure, which from the above derivation is apparently just the Jacobian $\mathrm{m}^{2}$; however, this term is not well defined, and it will be more thoroughly discussed later in this section.

Thermodynamic quantities can be calculated from here using well-known relations. As an example, the average size of the moment at temperature $T,\left\langle m_{i}(T)\right\rangle$, for a given energy

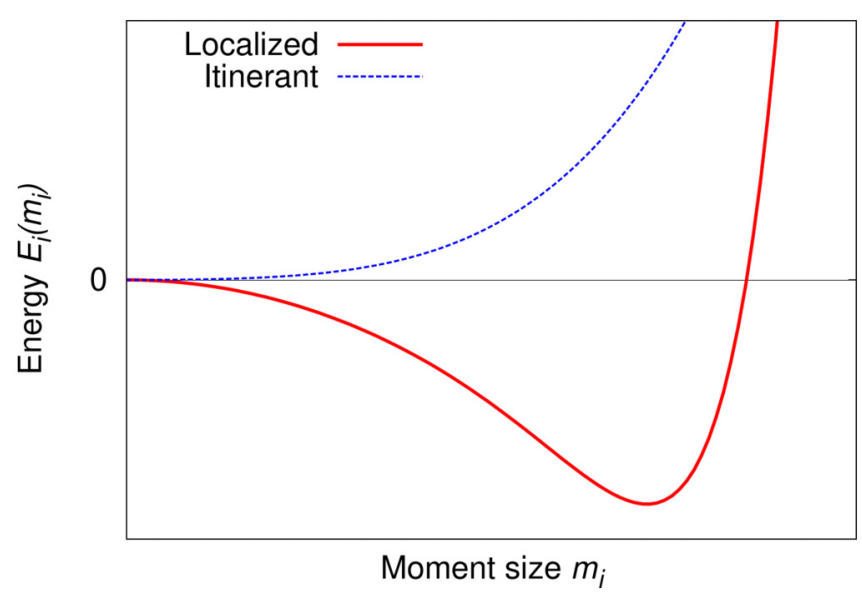

FIG. 1. Example of the typical energy landscapes for a localized moment (solid line) and an itinerant (dashed line) system in the paramagnetic state.

landscape $E_{i}$ is calculated as

$$
\left\langle m_{i}(T)\right\rangle=\frac{1}{Z_{i}} \int_{0}^{\infty} d m_{i} \operatorname{PSM} m_{i} e^{-\frac{E_{i}\left(m_{i}\right)}{k_{B} T}} .
$$

The partition function of a magnetic moment in a system in the paramagnetic state can be analytically calculated only for a quadratic form of the energy $E_{i}$ as a function of the moment size $m_{i}$; in this case, one can also derive an analytical expression for the magnetic entropy as a function of the average moment [31]. In the case of PSM $=m^{2}$ and considering a system in the paramagnetic state, this is $S^{\mathrm{mag}}=3 k_{b} \ln \langle m\rangle$, which differs from the entropy derived from the quantum Heisenberg model $S^{\mathrm{mag}}=k_{b} \ln (m+1)$, where LSFs are not considered. Schemes to include these analytical expressions of the magnetic entropy directly in the one-electron potentials in DFT calculations have also been developed [16,31,32]. However, a harmonic expression for the energy landscape is often very approximate; in fact, a fourth-order polynomial is more suited in both partly localized systems (such as bcc Fe, where this $S^{\text {mag }}$ would overestimate the magnetic moment) and itinerant ones (e.g., $\mathrm{Ni}$, where the moment is underestimated [37]). Therefore, numerical integration of the partition function needs to be performed in order to obtain thermodynamic properties of the system.

At this point, the issue of PSM should be addressed. First of all, different types of systems (localized moments or itinerant electrons) show different energy landscapes in the paramagnetic state (see Fig. 1), with the localized moment system having a minimum at $m \neq 0$, whereas the itinerant one has a minimum for $m=0$. Local moments in the itinerant system can be present only because of temperature. In general, at finite temperatures, the moments can evolve in time by rigid rotations (transversal fluctuations) and/or by changing their size (longitudinal fluctuations), where the former evolution is slower than the latter; the predominant mechanism is dictated by the energy landscape $E(m)$. For an itinerant system, assuming that at time $t=0$ a moment has value $m=\bar{m}$, the moment can change direction by shrinking down to zero and then reappearing in another direction. This evolution mechanism is fast compared to the rigid rotation of the moment, therefore 
coupling longitudinal and transversal DOFs. For this reason, the full dimensionality of the problem has to be taken into account when performing thermodynamic averages, and from here the use of PSM $=m^{2}$ is motivated. In contrast, for a localized moment system, the predominant mechanism of evolution is the rotation of the finite moment; therefore, the longitudinal fluctuations can be treated as adiabatically fast and confined to the direction of the moment, motivating the use of PSM $=1$ as required for a monodimensional problem. Intermediate PSMs have also been suggested [30]; however, no rigorous method to define which PSM should be used in which case is available. In this work, we do not aim at solving the problem of PSM, but we think that the reader should be aware of it, and we show the consequences of different choices of PSM in Sec. III A.

\section{B. Supercell scheme for calculation of magnetic moment size at finite temperature}

The calculation of energy landscapes like the ones shown in Fig. 1 is easily performed with the aid of constrained DFT calculations, and it can be carried out in both magnetically ordered and disordered states [25]. In this paper, we devise a scheme to calculate the landscapes at a given temperature which does not rely on symmetry in the system and is therefore applicable to a vibrating lattice or a system with, e.g., topological disorder like liquids.

Given $N$ atoms which, for simplicity, will all be considered to be magnetic, a particular atomic-magnetic configuration is defined by the positions of all atoms $\left\{\mathbf{R}_{1}, \ldots, \mathbf{R}_{N}\right\}$ and the direction and size of the moment associated with each of them $\left\{\mathbf{m}_{1}, \ldots, \mathbf{m}_{N}\right\}$ (for the source of this atomic-magnetic configuration, the reader is referred to Sec. II C). As an example, if the system has at least partly localized moments, a DFT calculation constraining only the direction of the moments along some preestablished direction will give the moment size that corresponds to $0 \mathrm{~K}$ temperature on the longitudinal DOFs (from now on referred to as " $0 \mathrm{~K}$ "), whereas for a system with " $0 \mathrm{~K}$ " nonmagnetic solution (itinerant), the initial magnetic configuration would have zero moments. The moments do not need to have the same magnitude even if the system involves only one element since we allow for disorder in atomic positions and moment direction; therefore, every moment experiences a unique environment.

Once the " $0 \mathrm{~K}$ " size of the moments is established, we proceed to calculate the energy landscape as a function of moment size for each atom in the supercell, which again can be different from each other due to the different local environments. To do this for a specific atom $i$, we fix the size and direction of all the other moments to the " $0 \mathrm{~K}$ " value previously calculated, and we perform completely constrained calculations (i.e., constraining both the direction and magnitude of the moments) varying only the size of moment $m_{i}$ in steps. We generally employ six different magnitudes of each moment $m_{i}$. Then, the specific energy landscape $E_{i}\left(m_{i}\right)$ is obtained by fitting a fourth-order polynomial as in Eq. (2) to the results of the constrained calculations for different values of $m_{i}$. An example of the energy vs moment size calculated in this way for moment $\mathbf{m}_{i}$ and the polynomial fit are shown in Fig. 2.

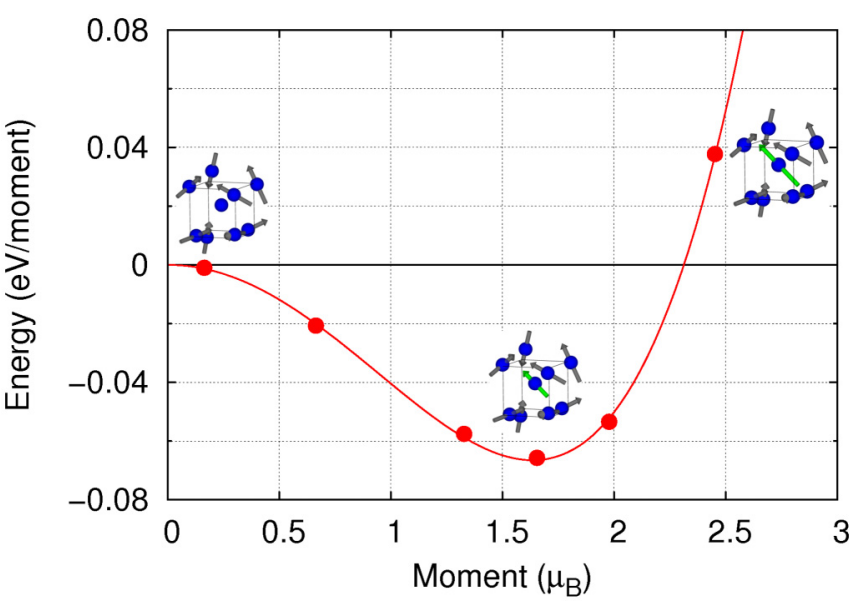

FIG. 2. Illustration of the procedure of the calculation of the energy landscape, where the energy points in the graph are calculated for different sizes of the moment on the central atom in the cartoons while keeping all the others fixed. This landscape is the actual landscape for one moment in bcc Fe at the Curie temperature (see Sec. III A).

This procedure is repeated for all atoms in the supercell, so that an energy landscape is obtained for each atom. The thermodynamic average moment of atom $i$ at a certain temperature $\bar{T},\left\langle m_{i}(\bar{T})\right\rangle$, calculated here numerically with Eq. (5), is chosen to be the moment of atom $i$ at $T=\bar{T}$. This average is just the pure thermodynamic average from Eq. (5), which does not involve an average over atoms in the supercell.

Since at this point the size of the moments at temperature $\bar{T}$ is different from the " $0 \mathrm{~K}$ " value, the energy landscapes $E_{i}$ might have changed. Therefore, the same procedure is reiterated for all the moments until $\left\langle m_{i}(\bar{T})\right\rangle$ does not considerably change compared to the previous iteration for each single atom, with a convergence criterion of $0.01 \mu_{B}$.

\section{Computational details and origin of the atomic configurations}

All calculations are carried out with the Vienna $\mathrm{Ab}$ initio Simulation Package (VASP) [38] employing projector augmented-wave potentials $[39,40]$ and the generalized gradient approximation with the Perdew-Burke-Ernzerhof functional [41] as the exchange and correlation functional. Supercells of 54 atoms $(3 \times 3 \times 3$ conventional bcc unit cells) are employed in the case of $\mathrm{Fe}$ at ambient pressure and $T \approx T_{C}=1043 \mathrm{~K}$ and bcc $\mathrm{Fe}$ at Earth's inner-core conditions ( $T \approx 6000 \mathrm{~K}, p \approx 300 \mathrm{GPa}$ ). For the liquid cases, we consider cubic boxes with 54 and 125 atoms for the highpressure and ambient-pressure cases, respectively. In the highpressure cases, the $3 s$ and $3 p$ electrons are considered valence electrons. All calculations are performed with noncollinear magnetism.

In Table I, the temperature, pressure, lattice parameter (for the solids), volume, and density of the studied cases are summarized.

For bcc Fe at ambient pressure, the atomic-magnetic configuration employed in the investigation is a snapshot of an ASD-AIMD run, performed with Langevin dynamics to 
TABLE I. Temperature, pressure, lattice parameter $a$, volume per atom $V$, and density $d$ of solid bcc and liquid (L) Fe considered in the present study. Pressures and temperatures reported here are approximate.

\begin{tabular}{lccccc}
\hline \hline & $T(\mathrm{~K})$ & $p(\mathrm{GPa})$ & $a(\AA)$ & $V\left(\AA^{3} /\right.$ atom $)$ & $d\left(\mathrm{~g} / \mathrm{cm}^{3}\right)$ \\
\hline bcc & $T_{C}$ & 0 & 2.882 & 11.97 & 7.74 \\
& 6000 & 300 & 2.410 & 7.00 & 13.24 \\
$\mathrm{~L}$ & $T_{m}$ & 0 & & 13.06 & 7.10 \\
& 6000 & 200 & & 7.81 & 11.87 \\
\hline \hline
\end{tabular}

control the temperature (damping parameter of $1 \mathrm{ps}^{-1}$ ), a cutoff energy for expansion of plane waves of $400 \mathrm{eV}$, and the first Brillouin zone sampled with a $\Gamma$-centered $2 \times 2 \times 2$ mesh for the AIMD part, evolving the atomic trajectories with a 1-fs time step. The direction of the moments is constrained with the method presented in Ref. [42], employing a constraining parameter $\lambda=10$. For what concerns the ASD part, which is performed with the UppASD code $[43,44]$, we employ a 0.01-fs time step for evolution of the moment direction and a damping parameter of 0.05 in the LLG equations. After equilibration, a combined atomic-magnetic configuration is taken to investigate LSFs. As previously mentioned, the " $0 \mathrm{~K}$ " moments are here obtained from a DFT calculation constraining only the direction of the moments. In this particular case, the direction of the moments is the one derived directly from the ASD-AIMD run since within this approach magnetic and atomic configurations are coupled.

Regarding bcc Fe at Earth's inner-core conditions, the snapshot is taken from a NM MD run, the results of which are reported in Ref. [36]. For the computational details regarding this simulation, the reader is referred to the original paper. In the calculation of LSFs in this case, we employ moments with random directions, with the first iteration performed for each atom in a NM background.

For ambient-pressure liquid $\mathrm{Fe}$, where the temperature chosen is around the melting point $\left(T_{m}=1823 \mathrm{~K}\right)$, the positions employed are obtained from a DLM-MD simulation[20]. In more detail, the simulation was started by placing 125 $\mathrm{Fe}$ atoms in a cubic box at random positions matching the experimental density at $T_{m}$. A NM run was then performed for $500 \mathrm{fs}$, and from these positions a DLM-MD simulation was carried out with a time step of $1 \mathrm{fs}$ while changing the collinear magnetic configuration every 5 fs. The total length of the simulation was about 6.5 ps. Fermi-Dirac smearing was included in the calculations, with the electronic temperature matching the melting temperature. The atomic configuration for the LSF analysis was randomly picked from the DLM-MD run. The details of the magnetic configuration are explained in Sec. III C.

The high-pressure liquid case, similar to high-pressure bcc $\mathrm{Fe}$, is investigated from a snapshot of a NM MD simulation at $T=6000 \mathrm{~K}$ and $p \approx 200 \mathrm{GPa}$, conditions expected in Earth's outer core.

The DFT calculations of the energy landscapes are performed with a thicker $k$-point mesh $(5 \times 5 \times 5$ MonkhorstPack mesh [45]) while constraining both the direction and size of the moments with the method implemented in VASP with $\lambda=25$.

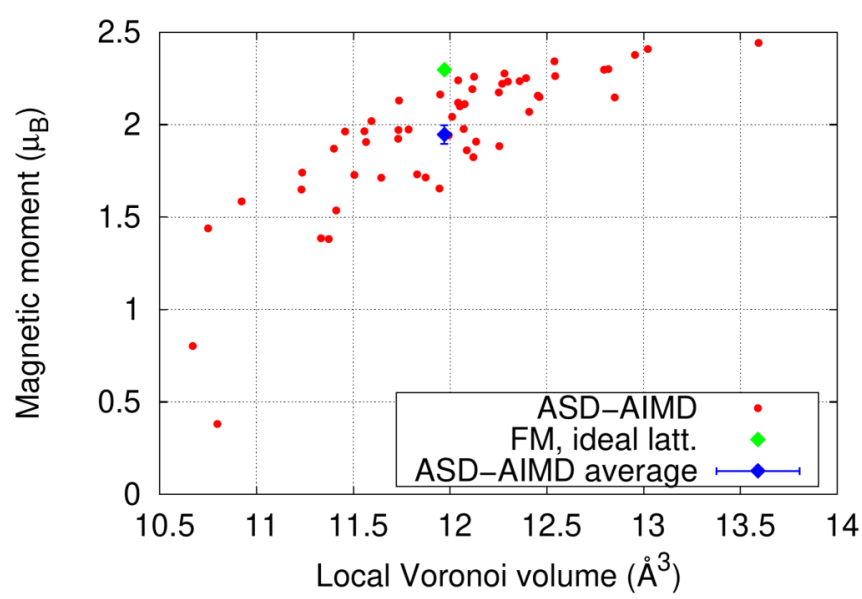

FIG. 3. Magnetic moments vs local Voronoi volume for bcc Fe from the ASD-AIMD simulation at the Curie temperature; a single value for each moment is obtained here because only the direction of the moments is constrained.

The error bars in the plots correspond to the error on the mean of the thermodynamic average moments (each obtained from a single landscape) and are defined as the standard error, i.e., the standard deviation of the distribution divided by the square root of the number of moments in the cell.

\section{RESULTS}

\section{A. bcc Fe at the Curie temperature}

As a starting point of the analysis, we calculate the " $0 \mathrm{~K}$ " size of the magnetic moments, shown in Fig. 3 as a function of the local Voronoi volume. As can be seen, the moment size shows a certain degree of correlation with the local Voronoi volume, with smaller moments for smaller volumes. The Voronoi volume and moment size for an ideal FM bcc lattice and the arithmetic average of the moments in the present configuration are also shown for comparison (green and blue diamonds, respectively). We observe that, as is already known [46], the average moment size is lower in this particular configuration compared to the FM moments on an ideal lattice.

At this point, the calculation of the energy landscapes for each moment $m_{i}$ is carried out according to the procedure described in Sec. II B. We calculate the effect of LSFs at $T=$ $1100 \mathrm{~K}$, and we employ both the monodimensional and threedimensional PSMs. In both cases, we reach convergence after three iterations. The fitted energy landscapes are shown in Fig. 4 for PSM $=m^{2}$; use of the other PSM gives differences in the landscapes, which is difficult to discern by eye.

As can be seen, the local environment affects strongly the shape of these moments, with two cases in which the landscapes are more similar to an itinerant rather than localized moment system, which is what bcc Fe is usually considered. These moments belong to atoms characterized by particularly small local Voronoi volumes. Of course, this is a special case; on average, during a simulation, one can expect to recover a more localized moment behavior. However, the dependence on the local environment affects the instantaneous forces, as will be shown later in this section, and therefore 


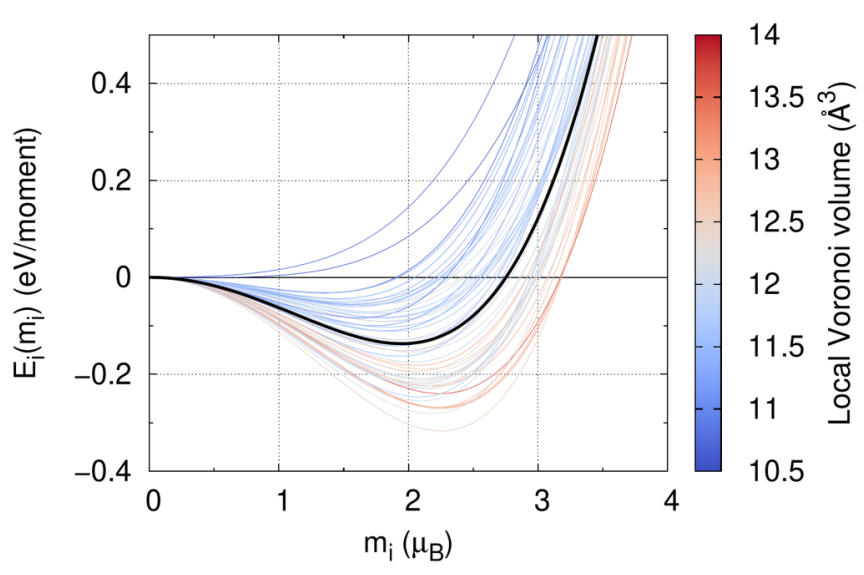

FIG. 4. Energy landscapes calculated with the present method for each atom in bcc $\mathrm{Fe}$ at the Curie temperature from the ASDAIMD snapshot with PSM $=m^{2}$. The color code indicates the size of the local Voronoi volume for the given atom. The black landscape indicates the average.

the dynamics. These effects might play an important role in the proper sampling of the phase space in, e.g., an AIMD simulation.

A further detail that should be noticed concerns the two moments with more itinerantlike behavior. As seen in Fig. 3, no moment actually has a zero value in the initial calculation with only the direction constrained; however, in the fully constrained calculations these two moments have minimum energy for $m=0$. This is probably due to the full constraint itself; that is, when we constrain only the direction of the moments, the system can rearrange the electrons to minimize the energy leading to nonzero moments, whereas with full constraint there is less room for rearrangement, and the lowest-energy solution now has $m=0$. Another reason for this discrepancy could be that the direct " $0 \mathrm{~K}$ " calculation gets stuck in a local minimum before reaching $m=0$ because of the numerics of the DFT code, which could happen for a very flat energy landscape.

The size of the moments with the inclusion of LSFs at temperature $T=1100 \mathrm{~K}$ at each iteration is shown in Fig. 5 for the different PSMs, and they are compared to the "0 K" value (the zeroth iteration). Between the first and second iterations, the moment size is generally already converged within $\approx 0.2 \mu_{B}$ for PSM $=m^{2}$, whereas for the monodimensional PSM the convergence is slower due to the larger differences in the size of most of the moments with LSF. In addition, it is interesting to notice that in the former case, LSFs induce an increment in the average moment size (black open diamonds in Fig. 5), whereas in the latter this quantity decreases. The average moment depicted here is obtained as the average of the moments, each obtained with its own energy landscape, at the given temperature; employment of the average landscape instead is different but gives a similar value with a small difference in the average moment $\left(<0.05 \mu_{B}\right)$.

The spread in landscapes due to local environments seems to suggest that bcc Fe cannot be treated as a fully localized moment system; therefore, PSM $=1$ is probably not the relevant PSM to consider. A certain degree of coupling between longitudinal and transversal DOFs would lead to

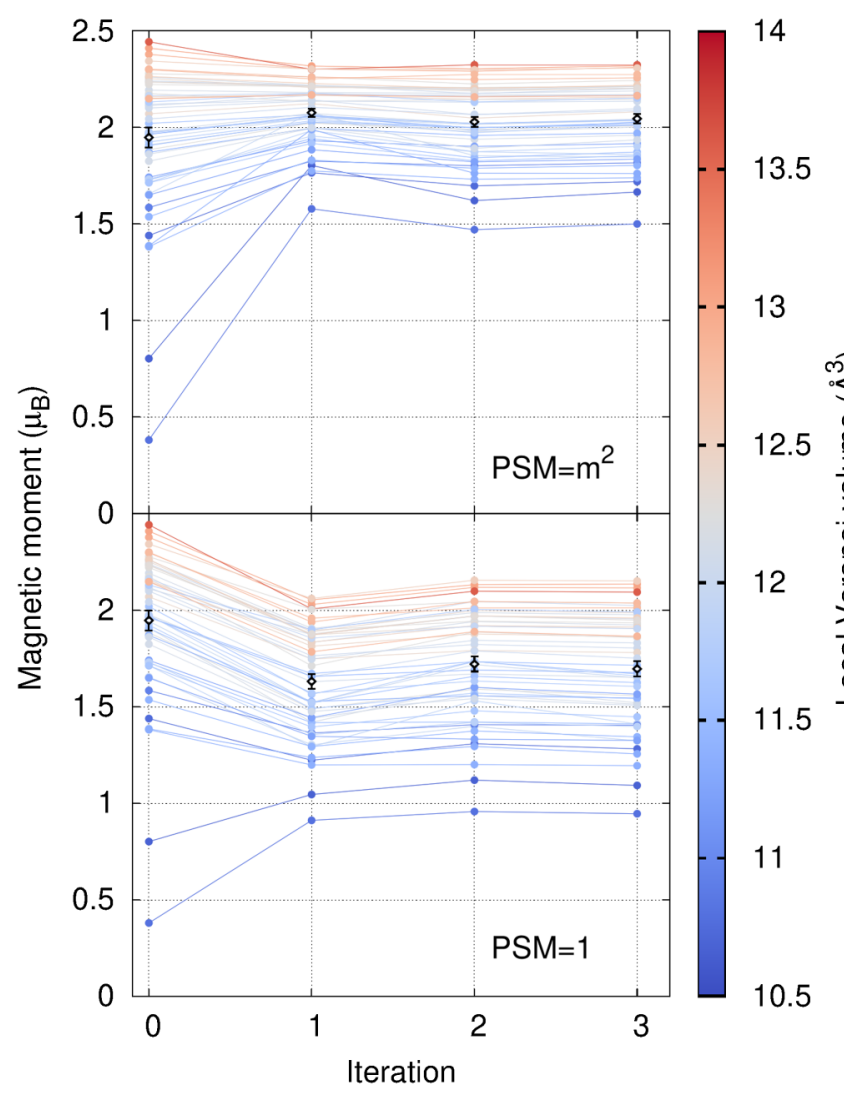

FIG. 5. Moment size with inclusion of LSFs at each iteration of the procedure for PSM $=m^{2}$ (top) and PSM $=1$ (bottom). The color of the lines and symbols is related to the size of the local Voronoi volume of the atom, whereas the open diamonds are the average moment at each iteration.

results intermediate between the present ones. An additional comment regarding PSM $=1$ concerns the limits of integration in the partition function: since in this case the problem is monodimensional, the integration space should be extended to negative values of the moments. In the present case of an even-power landscape, no difference would be found in average moment size; on the other hand, using a polynomial which includes odd powers would require fitting and integration in the negative-moment space. In any case, within the assumptions of the present study, this is not an issue. Finally, when using PSM $=1$, one needs to include the contribution to the entropy from the transverse DOFs outside the LSF scheme, e.g., by considering a Heisenberg model.

In order to estimate the effect of lattice and magnetic disorder on the energy landscapes, we perform the calculation of $E_{i}\left(m_{i}\right)$ with different degrees of disorder, starting from FM moments on an ideal bec lattice, then moving to the same instantaneous magnetic configuration (referred to as MSM) as that obtained from the ASD-AIMD simulation but on the ideal lattice, FM and MSM on atomic positions obtained from a FM-AIMD simulation, and, finally, the average landscape from Fig. 4. For these landscapes, we perform only one iteration of the procedure since already at this level of convergence conclusions on the effect of disorder can be inferred. In Fig. 6, 


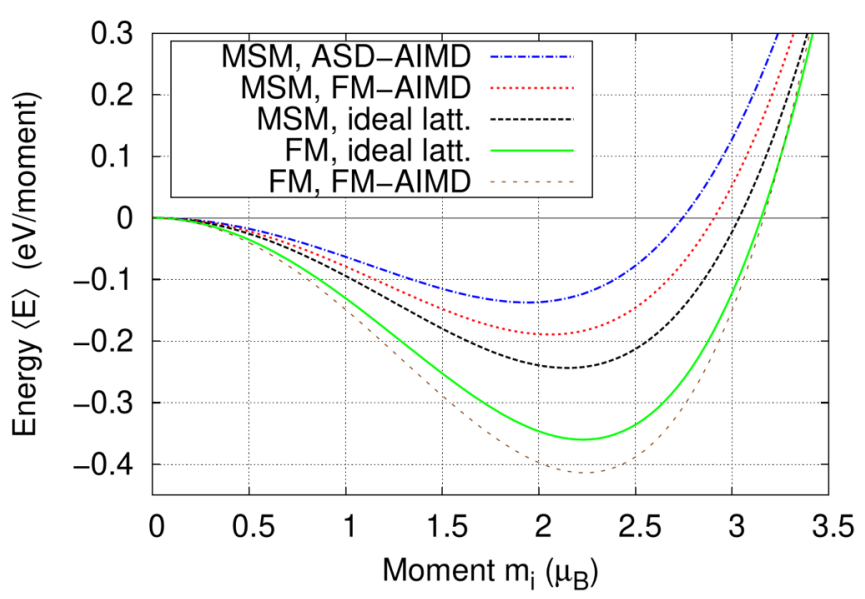

FIG. 6. Effect of different degrees of vibrational and magnetic disorder on the average energy landscape in bcc Fe. The magnetic sampling method (MSM) models the paramagnetic state.

the average landscapes for the different atomic-magnetic configurations are presented. It can immediately be seen that, keeping the FM state and introducing vibrational disorder, the landscape becomes deeper, which can be interpreted as a stronger localization of the moments. Changing the magnetic state to MSM on ideal-lattice positions leads to an increased itineracy in the system, represented here by shallower landscapes. In this state, the electronic structure is different from the FM state, and we observe that the introduction of higher degrees of lattice disorder leads to a higher degree of itineracy in the system, in contrast to the effect we observe for the FM state. Thus, the effects of magnetic and lattice disorder are not simply additive when it comes to the energy landscape for longitudinal spin fluctuations. It is worth mentioning again that these landscapes are given for a particular atomic-magnetic configuration with 54 atoms; therefore, if the procedure were performed on more configurations, a change in the quantitative values of the average landscapes could be expected. Nonetheless, we expect the qualitative picture to remain the same.

In this context, it is important to evaluate the effect of LSFs on the forces acting on the atoms, which will affect the evolution of the atomic positions during an AIMD simulation, therefore affecting the vibrational phase-space sampling. The change in intensity and direction of the forces with LSFs for the two different PSMs compared to the " $0 \mathrm{~K}$ " moment results is depicted in Fig. 7. Forces obtained with PSM = $m^{2}$ tend to be larger than the " $0 \mathrm{~K}$ " forces due to the general increase in magnetic moment size; for $\mathrm{PSM}=1$, the opposite happens [Fig. 7(a)]. The change in force modulus and direction is, in general, relatively small $(\approx 0.1 \mathrm{eV} / \AA$, $\left.0^{\circ}-5^{\circ}\right)$; however, for some atoms deviations are more important. Inclusion of LSFs also affects the pressure in the supercell, increasing by $\approx 1.5 \mathrm{GPa}$ with $\mathrm{PSM}=m^{2}$ and decreasing by $\approx 5 \mathrm{GPa}$ with PSM $=1$. The lattice parameter employed here is the $0 \mathrm{~K}$ theoretical lattice parameter expanded with the experimental thermal expansion coefficient. Since the " $0 \mathrm{~K}$ " atomic-magnetic configuration is at a pressure of $\approx-5 \mathrm{GPa}$, the inclusion of LSFs with PSM $=m^{2}$ seems to be an improvement.
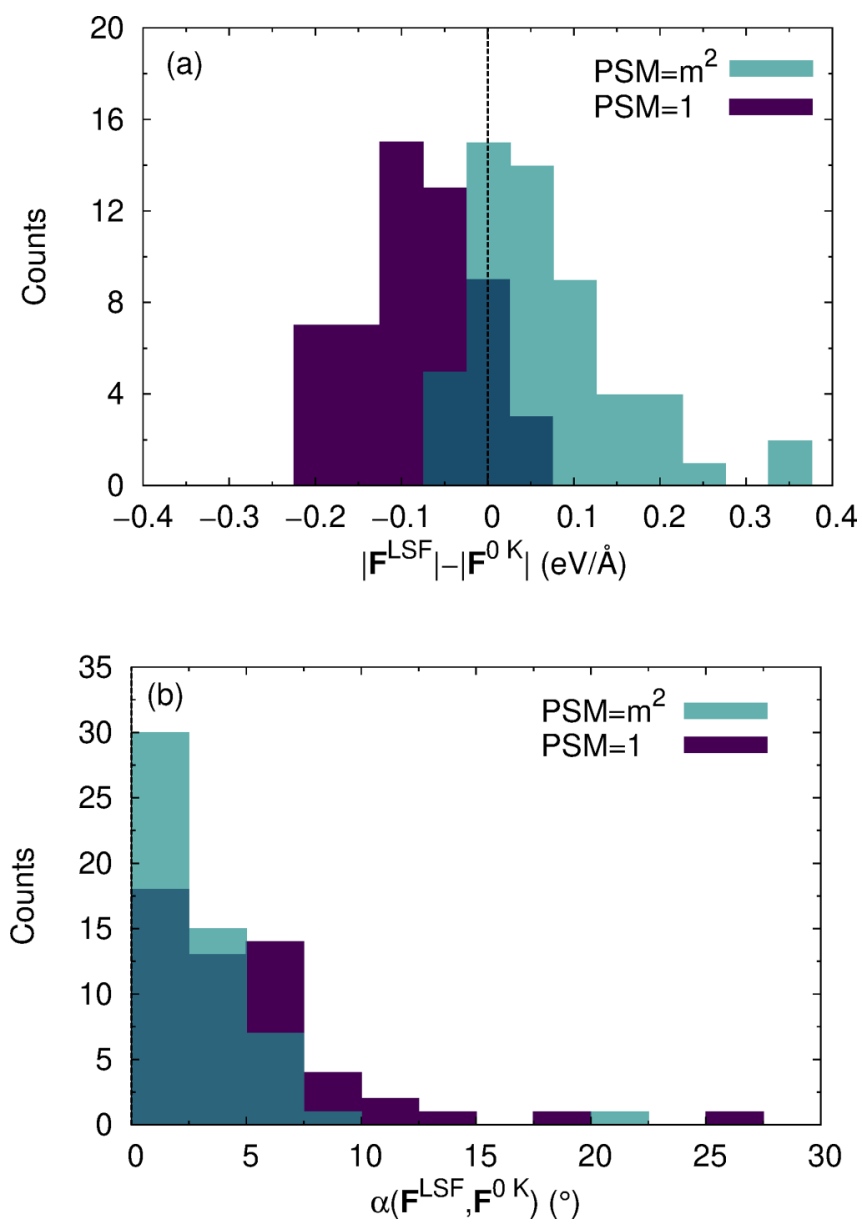

FIG. 7. (a) Difference in the modulus of the interatomic forces calculated with different PSMs and the " $0 \mathrm{~K}$ " value. (b) Angle between forces with and without inclusion of LSF.

\section{B. bcc Fe at Earth's inner-core conditions}

We move now to the investigation of the effect of LSFs in the case of bcc $\mathrm{Fe}$ at Earth's inner-core conditions, i.e., $T \approx 6000 \mathrm{~K}$ and $p \approx 300 \mathrm{GPa}$. It has been speculated [31,47] that magnetic effects might play an important role also at these extreme conditions, where local moments on the atoms would rise purely from LSFs. At these pressures, indeed, all moments die in an unconstrained DFT calculation, indicating a transition to itinerant moment behavior. Since the atomic positions in the present calculations are a snapshot of a NM MD simulation (54 atoms in the supercell, $V=7.0 \AA^{3} /$ atom) from Ref. [36], we start the procedure by considering a NM background, i.e., fixing the size of all moments other than the one under investigation to zero. The NM background is kept only for the first iteration. Convergence is reached after three iterations also in this case. We employ the three-dimensional PSM, motivated by the purely itinerant nature of the magnetic moments at these conditions. The electronic free energy at $T=6000 \mathrm{~K}$ is employed for the energy landscapes since the purely electronic degrees of freedom are supposed to be faster than the magnetic ones; in addition, employing electronic energy or free energy leads to differences only at a quantitative level in this case. 

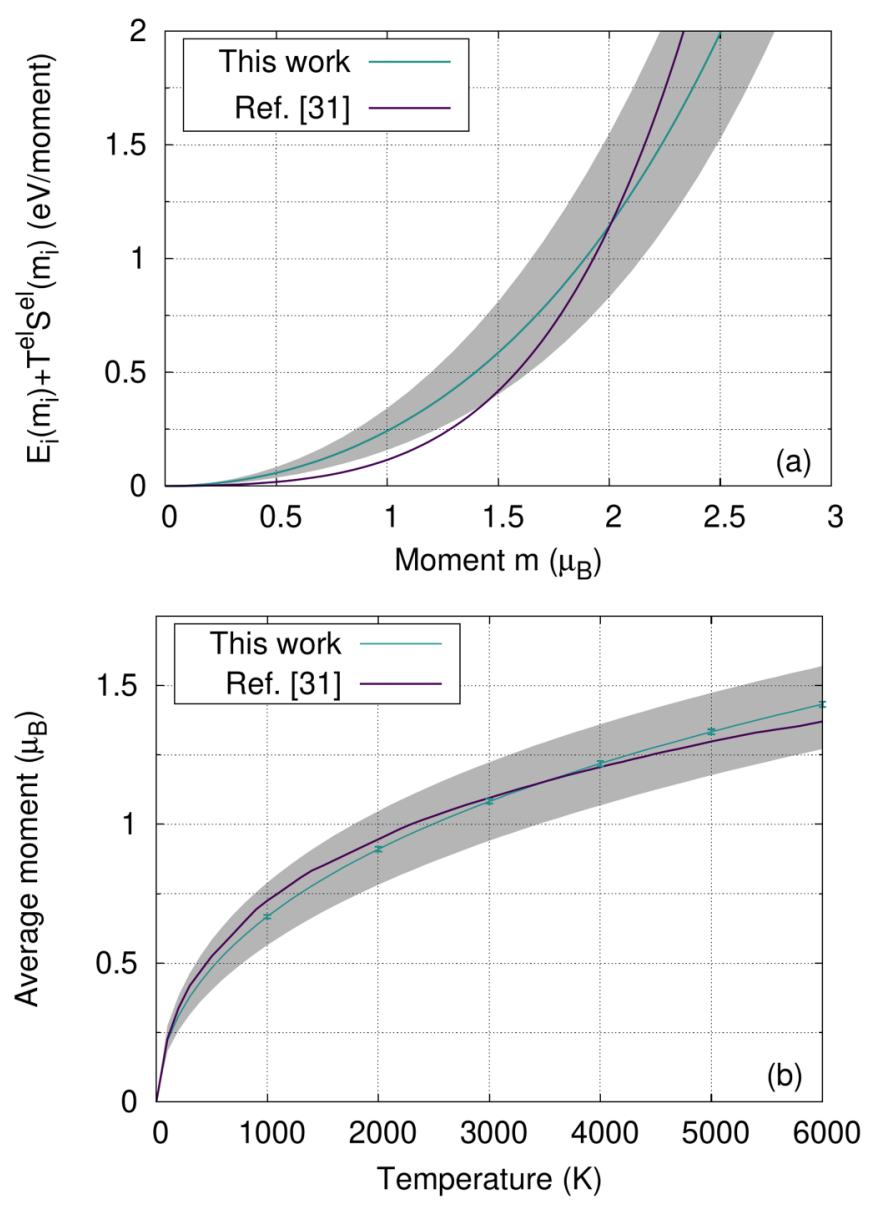

FIG. 8. (a) Average landscapes and (b) average moment as a function of LSF temperature for bcc Fe at Earth's inner-core conditions from the present work (cyan) compared to the one shown by Ruban et al. [31]. The gray area in both panels indicates the spread due to unique local environments for each moment. $T^{\mathrm{el}}$, the electronic temperature, is set to $6000 \mathrm{~K}$.

In Fig. 8(a) the average landscape obtained in the present work is compared to the landscape calculated by Ruban et al. [31], which was calculated on ideal-lattice positions. The colored area in Fig. 8(a) indicates the spread in landscapes due to atomic vibrations. The average landscape from the present work deviates from the one in Ref. [31]; however, no appreciable difference is found in the average moment as a function of temperature [Fig. 8(b)], with the two curves almost overlapping along the whole temperature range.

It is interesting to estimate the effect on forces derived from the employment of an average value of magnetic moment size for every atom at $6000 \mathrm{~K}$ (taken from Ref. [31]) compared to full consideration of local environment effects. In Fig. 9 we therefore compare the difference in forces between the NM calculation and the calculations with LSFs, both with the consideration of local environment effects and only on the mean level. The difference from the NM run is quite important, with values as large as $\approx 1 \mathrm{eV} / \AA$; however, the two LSF schemes give almost identical results, showing the small effect of individual local environment on the LSF moments and motivating, in this case, the use of the mean value for future investigations.

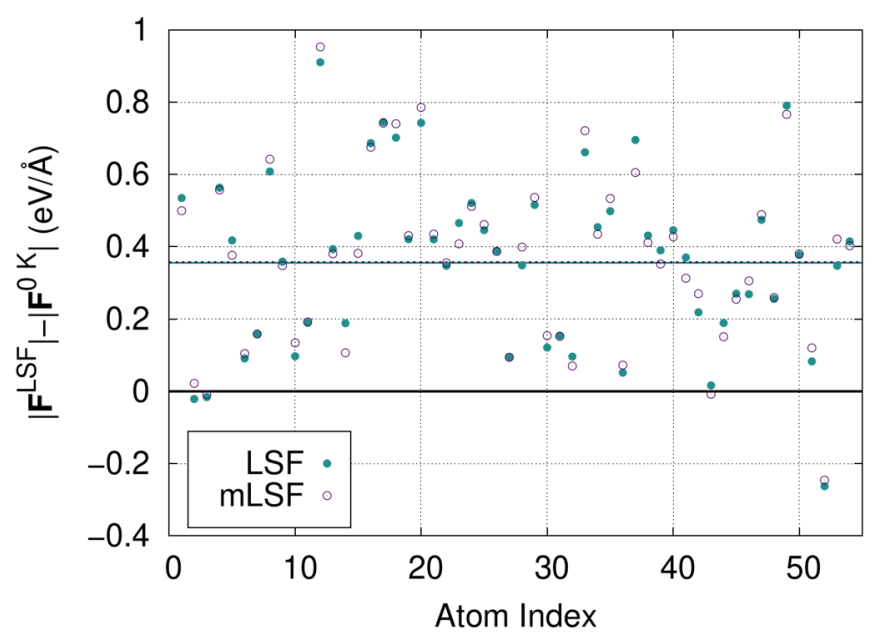

FIG. 9. Difference in the force modulus between inclusion and absence of LSF, both considering local environment effects (solid points) and including LSF only on a mean level (open points). The value of the moment at $6000 \mathrm{~K}$ on a mean level is taken from Ref. [31]. The average difference in forces is also depicted by horizontal lines, and the two lines for the different LSF schemes are superposed on each other.

This difference results also in a difference in total pressure in the cell. As previously mentioned, the volume used in the present investigation is $V=7.0 \AA^{3}$ /atom; for this volume, we obtain a pressure of $\approx 300 \mathrm{GPa}$ for the NM case, $320 \mathrm{GPa}$ with the inclusion of LSF on an average level, and $330 \mathrm{GPa}$ with the present self-consistent calculation of LSF moments.

Finally, the total electronic density of states (DOS) of bcc Fe at Earth's core conditions including vibrations with and without LSF is shown in Fig. 10. We observe a wellknown peak in the DOS near the Fermi level [47] for the NM calculation, which is here smooth due to the vibrational disorder in the lattice and the strong electron smearing; the inclusion of LSF leads to its complete disappearance. This

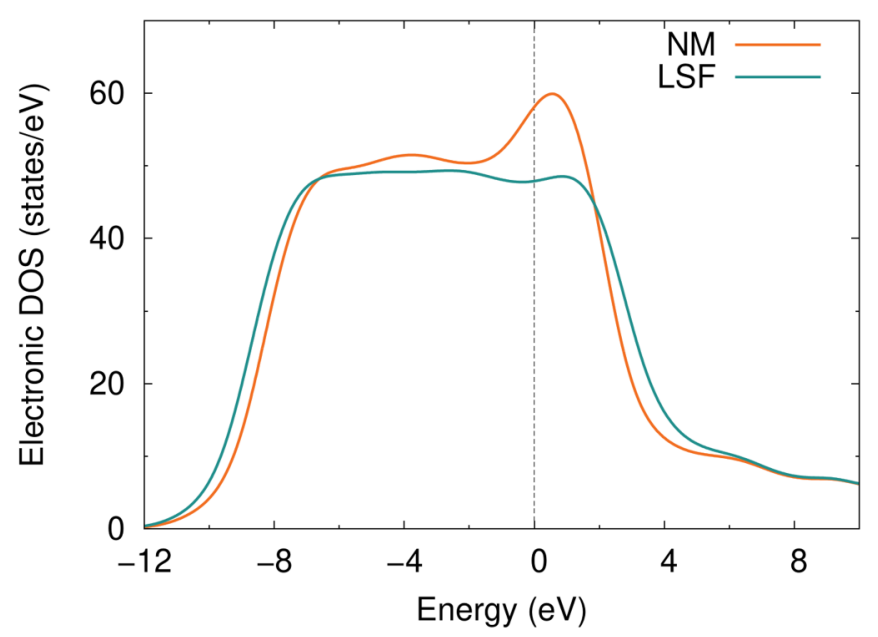

FIG. 10. Electronic DOS of bcc Fe at Earth's core conditions with and without LSF. Inclusion of LSF induces the complete disappearance of the peak around the Fermi level. 
effect is due to the fact that an increased atomic magnetization is achieved by splitting farther apart spin-up and spin-down states, inducing a reduction in the DOS at the Fermi level. A careful inspection of the consequences of this result is needed and is beyond the scope of the present investigation.

\section{Liquid Fe at ambient and high pressure}

The last examples of the power of the present scheme are concerned with liquid Fe at ambient and high $(\approx 200 \mathrm{GPa})$ pressure.

Starting from the ambient-pressure case, where the temperature employed is about the melting point, the atomic configuration is obtained as explained in Sec. IIC. Concerning the magnetic configuration at this stage of the analysis, noncollinear magnetic moments with random directions were assigned to each atom. Since the DFT calculation with noncollinear magnetic moments constrained only by direction does not converge, in order to get an initial guess of the size of the moments we calculate the energy landscape for ten atoms in a NM background. From the following iteration, we assign magnetic moments to every atom assuming a dependence on the Voronoi volumes. The choice of the ten atoms employed in the analysis is based on their Voronoi volumes, so that we are able to span the whole volume range available in the selected snapshot, which is, of course, larger than in the solid-state case. In the energy landscape we include also the electronic entropy contribution as in Sec. III B. We employ PSM $=m^{2}$ and perform three iterations to get the converged moments at $T=T_{m}$.

In Fig. 11 the energy landscapes and the magnetic moments with and without LSF are shown. The landscapes show a larger variety than the solid state counterpart because of the high degree of disorder in the liquid phase. LSFs tend to increase drastically the size of small moments (corresponding to small Voronoi volumes), whereas they leave roughly unchanged large moments. In particular, for the atom with a very large Voronoi volume $\left(\approx 17 \AA^{3}\right)$ we get an associated magnetic moment of size $\approx 3 \mu_{B}$, independent of the inclusion or absence of LSF, larger than any moment in bcc Fe.

Local magnetic moments are known to be present in liquid Fe from neutron scattering experiments [48], and they are found to be about $1.2 \mu_{B}$. This value is much smaller than the effective magnetic moment of $4.4 \mu_{B}$ obtained from the measurement of the magnetic susceptibility [49]. The discrepancy is attributed in Ref. [48] to the role played by conduction electrons in magnetic susceptibility measurements, which for more itinerant systems like fcc Fe and liquid $\mathrm{Fe}$ is assumed to be important. From our analysis of energy landscapes, we obtain average magnetic moments of $2.24 \mu_{B} \pm 0.13 \mu_{B}$ and $1.72 \mu_{B} \pm 0.32 \mu_{B}$ with and without inclusion of LSF, respectively. The latter value is obtained considering the size of the moments at the minimum of the energy landscapes. The average is weighted by the number of atoms with a Voronoi volume similar to the atoms for which the calculation of the magnetic moment is explicitly performed, so that moment sizes corresponding to frequent Voronoi volumes weigh more than moment sizes with rarer ones. Our results are consider-

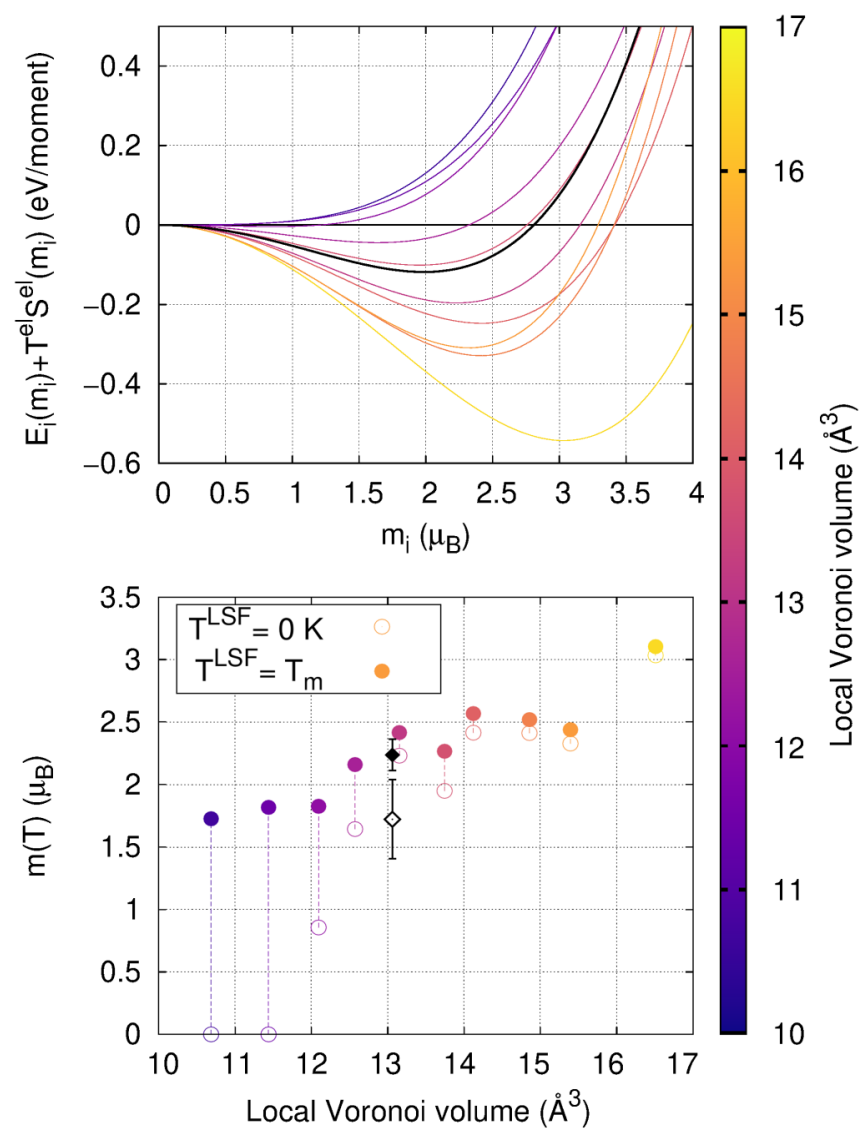

FIG. 11. Top: Free-energy landscapes calculated for selected atoms in liquid $\mathrm{Fe}$ around the melting point, where in the free energy the electronic entropy is included $\left(T^{\mathrm{el}}=T_{m}\right)$. Bottom: Moments with a LSF temperature of $0 \mathrm{~K}$ (open symbols) and equal to the melting temperature (solid symbols) for liquid $\mathrm{Fe}$ as a function of Voronoi volume. Diamonds indicate averages.

ably larger than the estimate from neutron scattering data both with and without the inclusion of LSF. However, in neutron scattering experiments at high temperatures it is difficult to separate magnetic and vibrational effects; therefore, the magnetic moment reported in Ref. [48] could be underestimated. In addition, in this work we have taken into account only one atomic-magnetic configuration, and the inherently disordered nature of liquids requires a much broader statistical sampling to obtain reliable results, which could then be compared to experiments or higher-level theories. This is clearly beyond the scope of the present analysis.

Concerning the case for Earth's outer-core conditions, the moments die off in a regular DFT calculation, which is what happens also to the solid at high pressure (see Sec. III B). In Fig. 12 the average free-energy landscape and average moment as a function of temperature are shown for this case, where PSM $=m^{2}$ is employed. As in the previous cases, the landscape is considered with the inclusion of the electronic entropic contribution at $T=6000 \mathrm{~K}$. We observe a less steep free-energy landscape compared to the solid-state case [compare with Fig. 8(a)], which results in larger moments at high temperature. Of course, this also has an effect on the pressure in the system, going from $\approx 190 \mathrm{GPa}$ in the 

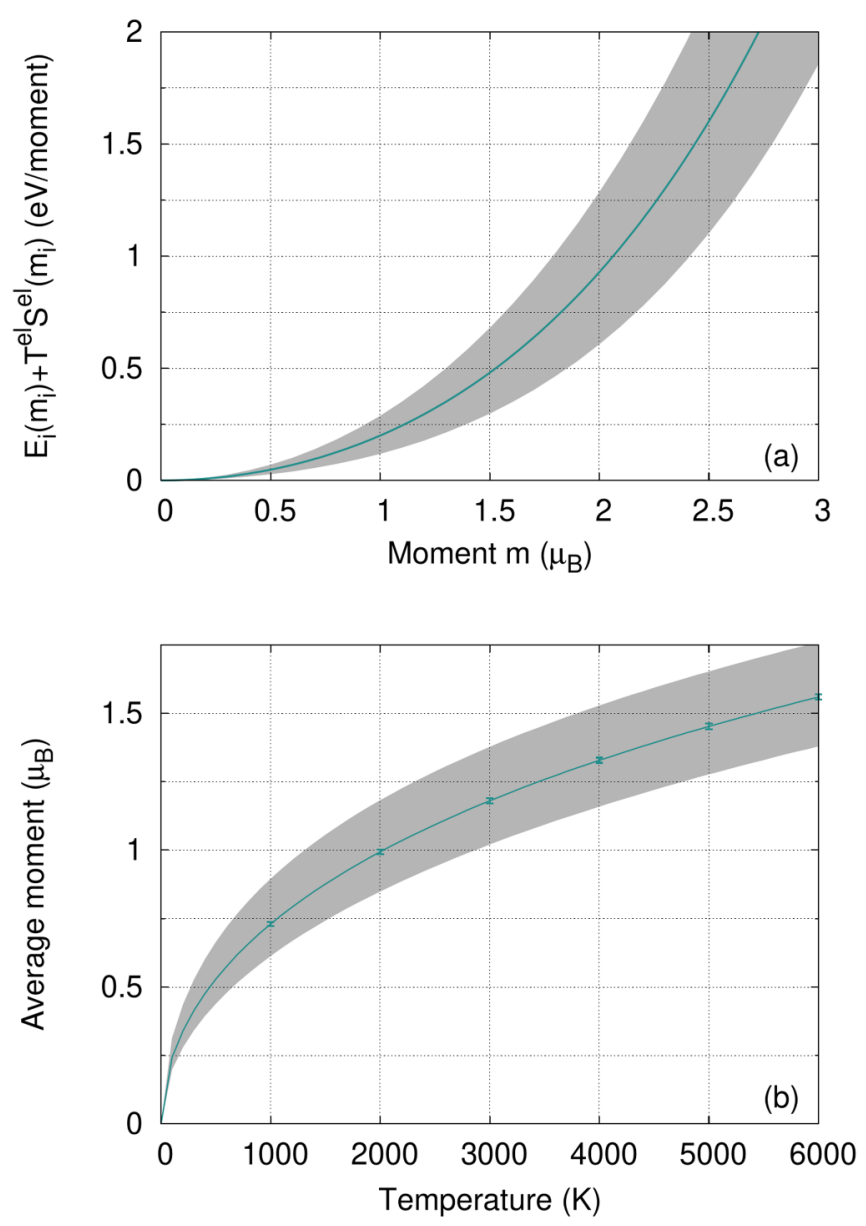

FIG. 12. (a) Average landscapes and (b) average moment as a function of LSF temperature for liquid $\mathrm{Fe}$ at Earth's outer-core conditions. The gray area in both panels corresponds to the spread due to unique local environments for each moment. As in Fig. 8(a), the electronic temperature is set to $6000 \mathrm{~K}$.

$\mathrm{NM}$ case to $\approx 220 \mathrm{GPa}$ in the case with LSF; the increase in pressure due to LSF is similar to the solid-state case. These results contradict the framework in which Ref. [50] was carried out, where a transition from paramagnetic to diamagnetic in liquid $\mathrm{Fe}$ was observed by collinear spinpolarized AIMD above $50 \mathrm{GPa}$. In that work, any contribution from LSF was neglected; therefore, the suggested transition is not supported by enough evidence. In the present work, we find that magnetic fluctuations could play a role even at larger temperatures and pressures than the ones considered in Ref. [50], including conditions of Earth's outer liquid core.

Recent experimental measurements of the equation of state of liquid $\mathrm{Fe}$ at high pressures and temperatures (up to $T=4350 \mathrm{~K}$ and $p=116 \mathrm{GPa}$ ) [51] suggest that at $p=220 \mathrm{GPa}$ and $T=5000 \mathrm{~K}$, the density of liquid $\mathrm{Fe}$ is $11.86 \mathrm{~g} / \mathrm{cm}^{3}$. In our calculation, the density is fixed to $11.87 \mathrm{~g} / \mathrm{cm}^{3}$, the temperature of the simulation from which the present snapshot is taken was $6000 \mathrm{~K}$, and the resulting pressure with LSF is $\approx 220 \mathrm{GPa}$, in good agreement with the experimental estimate. In contrast, at $5000 \mathrm{~K}$ and $190 \mathrm{GPa}$, which is the value of pressure we obtain without the inclusion of LSF, the experimentally estimated density is only $11.40 \mathrm{~g} / \mathrm{cm}^{3}$, suggesting that magnetic effects do play a role also at very high temperatures and pressures. In order to perform a full comparison with experiments, however, one should include also the kinetic virial pressure and carry out extremely accurate calculations to obtain fully converged stresses in the supercell. This goes beyond the scope of the present study; however, we underline the possible need to include LSFs in order to model appropriately such a complex system.

\section{CONCLUSIONS}

In this work we have developed a supercell approach for the derivation of the finite-temperature size of magnetic moments in magnetic materials based on the semiclassical theory of longitudinal spin fluctuations, which enables investigation of this degree of freedom in the presence of structural, vibrational, magnetic, and even liquid disorder in a self-consistent way. The work is carried out on the level of constrained noncollinear DFT calculations.

We found for bcc Fe at $T \approx T_{C}$ that lattice vibrations affect the on-site energy landscape as a function of moment size, leading to some more itinerant moments compared to the usual localized behavior; increasing the degree of disorder in the system makes the average energy landscape shallower. The choice of phase space measure is critical in this system since for the three-dimensional PSM the average magnetic moment increases compared to the " $0 \mathrm{~K}$ " size, whereas for the monodimensional PSM this quantity decreases. LSFs affect also force intensity and direction.

In the case of bcc Fe at Earth's inner-core conditions, we found that LSFs induce a substantial magnetic moment on the atom which strongly affects the forces in the system compared to nonmagnetic calculations. The present results are in good agreement with Ref. [31], suggesting that in this system LSFs can be included even at an average level, without considering local environment effects.

We performed the same analysis also for liquid Fe at ambient pressure and temperature about the melting point and at high pressure and temperature, conditions expected at Earth's outer core. In the former case, we found local moments also in " $0 \mathrm{~K}$ " calculations, which are then increased when LSFs are included in the analysis. In the latter, similar to the solidstate case for Earth's inner-core conditions, LSFs stabilize local magnetic moments, which can be very important for the geodynamics of Earth's outer liquid core.

\section{ACKNOWLEDGMENTS}

This research was carried out using computational resources provided by the National Supercomputer Centre (NSC) in Linköping (Sigma supercomputer) and the Swedish National Infrastructure for Computing (SNIC): Tetralith Cluster located at NSC. B.A. acknowledges financial support from the Swedish Research Council (VR) through International Career Grant No. 2014-6336 and Grant No. 2019-05403, from Marie Sklodowska Curie Actions, Cofund, Project INCA 600398, from the Swedish Government Strategic Research Area in Materials Science on Functional Materials 
at Linköping University (Faculty Grant SFOMatLiU No. 2009 00971), and from the Knut and Alice Wallenberg Foundation (Wallenberg Scholar Grant No. KAW-2018.0194), as well as support from the Swedish Foundation for
Strategic Research through the Future Research Leaders 6 program, FFL 15-0290. A. Ruban is gratefully acknowledged for useful discussions. D.G. thanks J. Klarbring for interesting discussions.
[1] R. McCallum, L. Lewis, R. Skomski, M. Kramer, and I. Anderson, Annu. Rev. Mater. Res. 44, 451 (2014).

[2] P. A. Claisse, in Civil Engineering Materials, edited by P. A. Claisse (Butterworth-Heinemann, Boston, 2016), pp. 321-337.

[3] R. Kuziak, R. Kawalla, and S. Waengler, Arch. Civ. Mech. Eng. 8, 103 (2008).

[4] L.-Q. Chen, L.-D. Chen, S. V. Kalinin, G. Klimeck, S. K. Kumar, J. Neugebauer, and I. Terasaki, npj Comput. Mater. 1, 15007 (2015).

[5] W. Counts, M. Friák, D. Raabe, and J. Neugebauer, Acta Mater. 57, 69 (2009).

[6] D. M. Edwards and E. P. Wohlfarth, Proc. R. Soc. London, Ser. A 303, 127 (1968).

[7] T. Moriya, Spin Fluctuations in Itinerant Electron Magnetism, Springer Series in Solid-State Sciences (Springer, Berlin, 1985).

[8] N. Melnikov and B. Reser, Dynamic Spin-Fluctuation Theory of Metallic Magnetism (Springer, Berlin, 2018).

[9] I. Abrikosov, A. Ponomareva, P. Steneteg, S. Barannikova, and B. Alling, Curr. Opin. Solid State Mater. Sci. 20, 85 (2016).

[10] N. B. Melnikov and B. I. Reser, Phys. Met. Metallogr. 117, 1328 (2016).

[11] L. V. Pourovskii, B. Amadon, S. Biermann, and A. Georges, Phys. Rev. B 76, 235101 (2007).

[12] P. Delange, T. Ayral, S. I. Simak, M. Ferrero, O. Parcollet, S. Biermann, and L. Pourovskii, Phys. Rev. B 94, 100102(R) (2016).

[13] A. V. Ruban, S. Shallcross, S. I. Simak, and H. L. Skriver, Phys. Rev. B 70, 125115 (2004).

[14] J. Yin, M. Eisenbach, D. M. Nicholson, and A. Rusanu, Phys. Rev. B 86, 214423 (2012).

[15] A. Lindmaa, R. Lizárraga, E. Holmström, I. A. Abrikosov, and B. Alling, Phys. Rev. B 88, 054414 (2013).

[16] A. V. Ruban and O. E. Peil, Phys. Rev. B 97, 174426 (2018).

[17] M. Pajda, J. Kudrnovský, I. Turek, V. Drchal, and P. Bruno, Phys. Rev. B 64, 174402 (2001).

[18] B. L. Gyorffy, A. J. Pindor, J. Staunton, G. M. Stocks, and H. Winter, J. Phys. F 15, 1337 (1985).

[19] B. Alling, T. Marten, and I. A. Abrikosov, Phys. Rev. B 82, 184430 (2010).

[20] P. Steneteg, B. Alling, and I. A. Abrikosov, Phys. Rev. B 85, 144404 (2012).

[21] I. Stockem, A. Bergman, A. Glensk, T. Hickel, F. Körmann, B. Grabowski, J. Neugebauer, and B. Alling, Phys. Rev. Lett. 121, 125902 (2018).

[22] K. K. Murata and S. Doniach, Phys. Rev. Lett. 29, 285 (1972).

[23] M. Uhl and J. Kübler, Phys. Rev. Lett. 77, 334 (1996).

[24] N. M. Rosengaard and B. Johansson, Phys. Rev. B 55, 14975 (1997).
[25] A. V. Ruban, S. Khmelevskyi, P. Mohn, and B. Johansson, Phys. Rev. B 75, 054402 (2007).

[26] L. M. Sandratskii, Phys. Rev. B 78, 094425 (2008).

[27] A. L. Wysocki, J. K. Glasbrenner, and K. D. Belashchenko, Phys. Rev. B 78, 184419 (2008).

[28] Z. Dong, W. Li, D. Chen, S. Schönecker, M. Long, and L. Vitos, Phys. Rev. B 95, 054426 (2017).

[29] F. Pan, J. Chico, A. Delin, A. Bergman, and L. Bergqvist, Phys. Rev. B 95, 184432 (2017).

[30] S. Khmelevskyi, J. Magn. Magn. Mater. 461, 14 (2018).

[31] A. V. Ruban, A. B. Belonoshko, and N. V. Skorodumova, Phys. Rev. B 87, 014405 (2013).

[32] H. Ehteshami and P. A. Korzhavyi, Phys. Rev. B 96, 224406 (2017).

[33] I. Leonov, A. I. Poteryaev, Y. N. Gornostyrev, A. I. Lichtenstein, M. I. Katsnelson, V. I. Anisimov, and D. Vollhardt, Sci. Rep. 4, 5585 (2014).

[34] Q. Han, T. Birol, and K. Haule, Phys. Rev. Lett. 120, 187203 (2018).

[35] N. B. Melnikov, G. V. Paradezhenko, and B. I. Reser, Theor. Math. Phys. 201, 1531 (2019).

[36] A. B. Belonoshko, T. Lukinov, J. Fu, J. Zhao, S. Davis, and S. I. Simak, Nat. Geosci. 10, 312 (2017).

[37] A. V. Ruban, Phys. Rev. B 95, 174432 (2017).

[38] G. Kresse and J. Hafner, Phys. Rev. B 47, 558 (1993); 49, 14251 (1994); G. Kresse and J. Furthmüller, Comput. Mater. Sci. 6, 15 (1996); Phys. Rev. B 54, 11169 (1996).

[39] P. E. Blöchl, Phys. Rev. B 50, 17953 (1994).

[40] G. Kresse and D. Joubert, Phys. Rev. B 59, 1758 (1999).

[41] J. P. Perdew, K. Burke, and M. Ernzerhof, Phys. Rev. Lett. 77, 3865 (1996).

[42] P.-W. Ma and S. L. Dudarev, Phys. Rev. B 91, 054420 (2015).

[43] B. Skubic, J. Hellsvik, L. Nordström, and O. Eriksson, J. Phys.: Condens. Matter 20, 315203 (2008).

[44] O. Eriksson, A. Bergman, L. Bergqvist, and J. Hellsvik, Atomistic Spin Dynamics: Foundations and Applications (Oxford University Press, Oxford, 2017).

[45] H. J. Monkhorst and J. D. Pack, Phys. Rev. B 13, 5188 (1976).

[46] B. Alling, F. Körmann, B. Grabowski, A. Glensk, I. A. Abrikosov, and J. Neugebauer, Phys. Rev. B 93, 224411 (2016).

[47] L. V. Pourovskii, T. Miyake, S. I. Simak, A. V. Ruban, L. Dubrovinsky, and I. A. Abrikosov, Phys. Rev. B 87, 115130 (2013).

[48] Y. Waseda and K. Suzuki, Phys. Status Solidi B 39, 669 (1970).

[49] G. Urbain and E. Übelacker, Adv. Phys. 16, 429 (1967).

[50] J.-A. Korell, M. French, G. Steinle-Neumann, and R. Redmer, Phys. Rev. Lett. 122, 086601 (2019).

[51] Y. Kuwayama, G. Morard, Y. Nakajima, K. Hirose, A. Q. R. Baron, S. I. Kawaguchi, T. Tsuchiya, D. Ishikawa, N. Hirao, and Y. Ohishi, Phys. Rev. Lett. 124, 165701 (2020). 